\title{
Who cares about the earthquake? Towards the the next generation of warning systems
}

\section{Diego Melgar}

University of Oregon (dmelgarm@uoregon.edu)

The quantity of interest in earthquake and tsunami warning systems is the hazard. This is either the intensity of shaking or the expected tsunami amplitude at a location of interest. Traditionally, earthquake and tsunami warning systems have relied on a rapid characterization of the earthquake, it's location, magnitude, dimensions, amount of slip, etc. From this information empirical relationships or rapid models are used to derive the implied shaking or tsunami amplitude. With the worldwide proliferation of dense on-shore and off-shore geophysical networks with diverse instrumentation it is becoming increasingly possible to eschew that paradigm for a modern approach. In this talk we will discuss recent work that shows how direct characterization of the earthquake hazards is possible by leveraging these new networks and modern data science techniques such as machine learning and data assimilation methods. In the early warning systems of the future knowledge of the particular details of the earthquake rupture process takes a back seat to direct estimation of the expected hazards. 